\title{
Application of Fuzzy Comprehensive Evaluation on COGAG Power Plant of Performance
}

\author{
Menglin Shao', Qianchao Liang1, Dong Yan', Heng Qin², Jun Xiang2 \\ ${ }^{1}$ The College of Naval, University of Engineering, Wuhan, China \\ ${ }^{2}$ The DaLain Naval Vessel Academy, Dalian, China \\ Email: smlin18@163.com
}

Received May 2014

\begin{abstract}
A fuzzy comprehensive evaluation of COGAG power plant performance is given by using a method of fuzzy mathematics, and multilevel fuzzy evaluation model is set up. Taking a naval ship as an example, the major parameters related to COGAG power plant performance are obtained by computer simulation, and a set of synthetic performance evaluation index system is established. The result shows that the strong fuzzy propulsion system performance indexes can be quantified by this fuzzy evaluation model in order to provide the reference for choosing the optimal design of ship propulsion system.
\end{abstract}

\section{Keywords}

COGAG Power Plant, Fuzzy Comprehensive Evaluation, Performance Index

\section{Introduction}

COGAG power plant [1] [2] will have a broad application prospect in the future, because its excellent maneuver capability, high single power density and adaptation for large range working condition. The propulsion system consists of four gas turbines (GT), two gearboxes, and two controllable pitch propellers (CPP). Two shaft generators are directly mounted on the shafts. The ship is able to operate in several propulsion modes: one to four gas turbines, one or two shaft lines, etc. [3]. The performance of power plant directly affects the economy and mobility of entire ship so that evaluating its performance could provide referential evidence when applied to design other type of power plant. On basis of fuzzy comprehensive evaluation, this paper establishes multilevel fuzzy evaluation model of COGAG power plant by depending on some major parameters obtained by computer simulation. With this model, the performance of COGAG power plant is analyzed to offer data for the users and decision-makers.

\section{Principle of Fuzzy Comprehensive Evaluation Method}

Fuzzy comprehensive evaluation method is a synthetic evaluation method based on fuzzy mathematics [4]. The

How to cite this paper: Shao, M.L., Liang, Q.C., Yan, D., Qin, H. and Xiang, J. (2014) Application of Fuzzy Comprehensive Evaluation on COGAG Power Plant of Performance. Journal of Power and Energy Engineering, 2, 29-34. 
evaluation method changes the qualitative evaluation into quantitative one according to the principle of membership degree in fuzzy mathematics. The fuzzy comprehensive evaluation method is suitable for solving Nondeterministic problems because of its features, such as clearer result, systematic approach and making solutions about fuzzy and difficult-quantized problem. Fuzzy synthetic evaluation model is mainly made up of singlelevel fuzzy comprehensive evaluation model and multi-level fuzzy comprehensive evaluation model [5].

\subsection{Single-Level Fuzzy Evaluation Model}

The 3 elements of the comprehensive evaluation: factors set, remark set, single factor evaluation set. Factors set $U=\left\{u_{1}, u_{2} \ldots, u_{n}\right\}$, the set of factors about the object of being judged. Remark set $V=\left\{v_{1}, v_{2} \ldots, v_{m}\right\}$, the set of comments. Single factor evaluation set means the evaluation of single factor in $U_{i}(i=1,2, \ldots, n)$, and gets the fuzzy set $\left(r_{i 1}, r_{i 2}, \ldots, r_{i m}\right)$ in $V$, which is a fuzzy mapping from $U$ to $V$, thus the fuzzy set $\left(r_{i 1}, r_{i 2}, \ldots, r_{i m}\right)$ in $V$ can be got.

$$
\begin{gathered}
f: U \rightarrow F(V) \\
u_{i} \mapsto\left(r_{i 1}, r_{i 2}, \cdots, r_{i m}\right)
\end{gathered}
$$

Fuzzy mapping $f$ can determine a fuzzy relationship $R \in \mu_{n \times m}$, called evaluation matrix, which is composed of single factor fuzzy sets for evaluation.

$$
R=\left[\begin{array}{llll}
r_{11} & r_{12} & \cdots & r_{1 m} \\
r_{21} & r_{22} & \cdots & r_{2 m} \\
\cdots & \cdots & \cdots & \cdots \\
r_{n 1} & r_{n 2} & \cdots & r_{n m}
\end{array}\right]
$$

Because of the differences of each single factor, all factors are needed to be weighted; using fuzzy set $A=\left(a_{1}, a_{2}, \ldots, a_{n}\right)$ in $U$ represents the weight distribution of each factor. As Equation (1) shows, the comprehensive evaluation for each factor is composed fuzzy set $A$ with evaluation matrix $R$.

$$
B=A \circ R
$$

\subsection{Multi-Level Fuzzy Evaluation Model}

In complex system, satisfactory result can't be obtained easily by using 1st-level fuzzy comprehensive evaluation model when the factors of the evaluation object are more than usual, where exists different levels between each factor. Evaluation factors should be classified due to the characteristics of evaluation object, and evaluated synthetically level by level, which is multi-level fuzzy comprehensive evaluation. The steps of multi-level fuzzy comprehensive evaluation are as follows:

- Divide the evaluation factors set $U$ into $m$ subsets according to the attributes, which meets Equation (2):

$$
\sum_{i=1}^{m} U_{i}=U,\left\{U_{i} \cap U_{j}=\Phi(i \neq j)\right\}
$$

- Pre According to Equation (2), the second-level evaluation factors set $U=\left\{U_{1}, U_{2}, \cdots, U_{m}\right\}$ can be got. $U_{i}=\left\{U_{i k}\right\} \quad\left(i=1,2, \ldots, m, k=1,2, \ldots, n_{k}\right)$ means $n_{k}$ evaluation factors in the subset $U_{i}$, which could be evaluated by using the single-level fuzzy comprehensive evaluation model. If the weight distribution in $U_{i}$ is $A_{i}$, the corresponding evaluation matrix is $R_{i}$. The comprehensive evaluation result of the $i$-th subset $U_{i}$ can be got as Equation (3):

$$
B_{i}=A_{i} \circ R_{i}=\left[b_{i 1}, b_{i 2}, \cdots, b_{i n}\right]
$$

- Evaluate synthetically for the $m$ evaluation factors subsets $U_{i}$ in $U$, the decision matrix is $R$ (see Equation (4)).

$$
R=\left[\begin{array}{c}
B_{1} \\
B_{2} \\
\vdots \\
B_{m}
\end{array}\right]=\left[\begin{array}{cccc}
b_{11} & b_{12} & \cdots & b_{1 n} \\
b_{21} & b_{22} & \cdots & b_{2 n} \\
\vdots & \vdots & \vdots & \vdots \\
b_{m 1} & b_{m 2} & \cdots & b_{m n}
\end{array}\right]
$$


If weight of each evaluation factors subsets in $U$ is $A$, the result of evaluation is $B=A \circ R$. Similarly, Construct third-level, fourth-level fuzzy synthetic evaluation model could be worked out. Figure 1 shows the sketch map of second-level fuzzy synthetic evaluation model.

In conclusion, the $(U, V, R)$ forms a fuzzy comprehensive evaluation mathematics model. The fuzzy transform $R$ in which change fuzzy subset $U_{i}$ in $\mathrm{U}$ to fuzzy subset $B$ in $V$. The $B$ is a fuzzy comprehensive evaluation to the evaluation object.

\section{Application Example}

\subsection{Performance Index Framework Analysis}

On the base of the principle of maximum membership degree and fuzzy linear transformation, main relevant indexes are taken into consideration to make a reasonable assessment, which is the basic idea of fuzzy comprehensive evaluation. The performance indexes of power plant are determined by the type, primary skill parameters and driving methods of engine [6]. For COGAG power plant, the performance evaluation indexes are divided into 3 layers by screening.

The following are estimate index of each level:

- The target layer: Comprehensive performance evaluating system of COGAG power plant.

- The first-degree index: 1) Economy; 2) Flexibility; 3) Concealment; 4) Reliability; 5) Repairable.

- The second-degree index: As Figure 2 shows, there are 22 indexes corresponded with first-degree index.

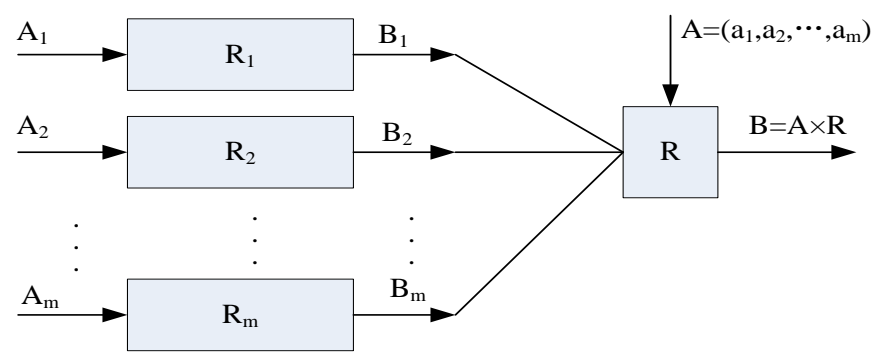

Figure 1. The sketch map of second-level Fuzzy comprehensive evaluation model.

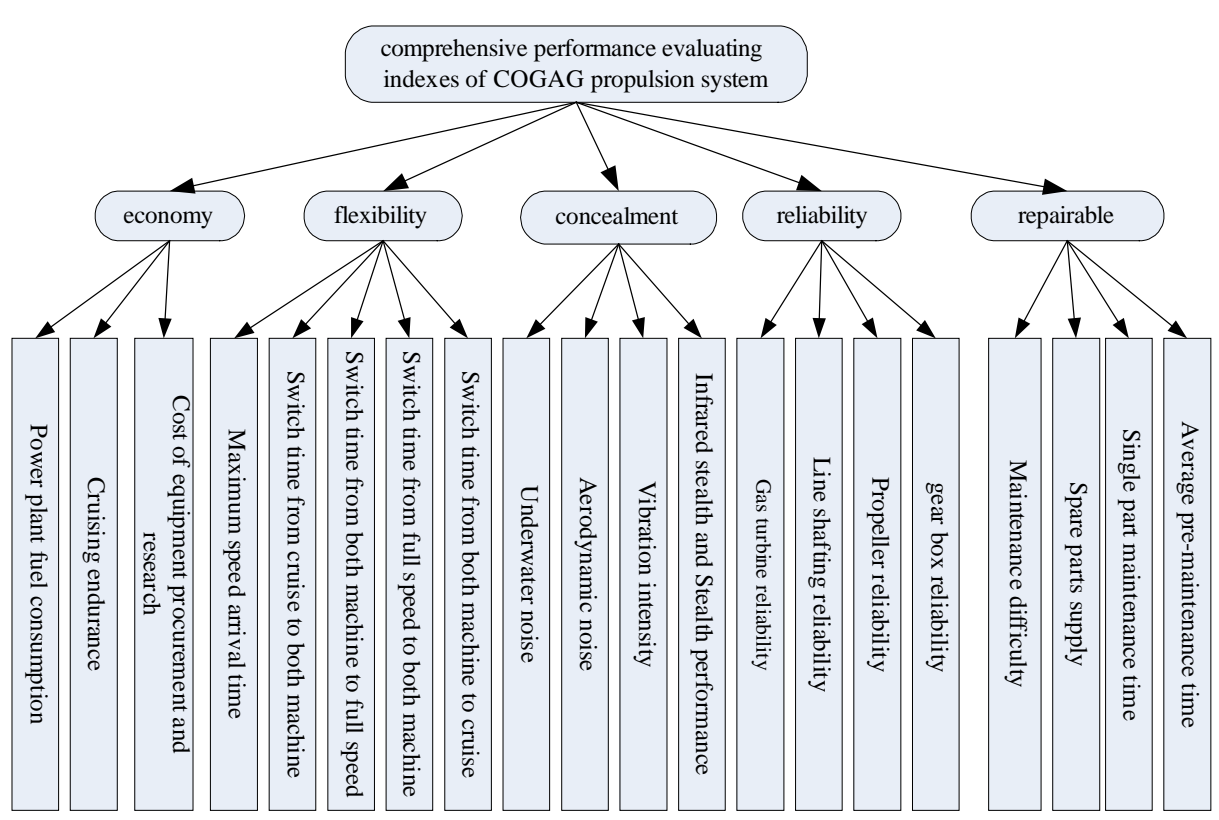

Figure 2. The sketch map of multilevel performance evaluation index of COGAG power plant. 


\subsection{Index Weight}

The major parameters related to COGAG power plant performance are obtained by simulation. Then, the involved factors are graded according to experts grading method, thus the weight of each index is achieved [7]. With a view to the difference from invited experts, 3 groups are divided, which have been given corresponding weight. The specific data can be seen from Table 1. Table 2 displays Weight for performance indexes and mean for evaluation of all experts.

Table 1. Subgroup of experts and weight distribution.

\begin{tabular}{clcc}
\hline Subgroup & Expert & Number & Weight \\
\hline \multirow{2}{*}{1} & Expert of Ship Design Department & 2 & $0.079 / 0.077$ \\
& Senior Engineer & 1 & 0.078 \\
& Researcher & 2 & $0.075 / 0.062$ \\
& Academic Expert & 1 & 0.063 \\
\cline { 2 - 3 } & Professor & 2 & $0.067 / 0.061$ \\
& Vice-professor & 1 & 0.062 \\
& Senior Engineer of Grass-root Units & 2 & $0.064 / 0.061$ \\
& Chief Engineer of Warship & 3 & $0.058 / 0.059 / 0.060$ \\
& Military Officer & 1 & 0.074 \\
\hline
\end{tabular}

Table 2. Weight for performance indexes and mean for evaluation of all experts.

\begin{tabular}{|c|c|c|c|c|}
\hline First-degree index & Weight & Second-degree index & Weight & Mean \\
\hline \multirow{4}{*}{ Economy } & \multirow{4}{*}{0.34} & Power plant fuel consumption & 0.33 & 0.81 \\
\hline & & Cruising endurance & 0.37 & 0.93 \\
\hline & & Cost of equipment procurement & 0.14 & 0.65 \\
\hline & & Cost of equipment research & 0.16 & 0.73 \\
\hline \multirow{5}{*}{ Flexibility } & \multirow{5}{*}{0.24} & Maximum speed arrival time & 0.24 & 0.88 \\
\hline & & Switch time from cruise to both machine & 0.23 & 0.76 \\
\hline & & Switch time from both machine to full speed & 0.28 & 0.58 \\
\hline & & Switch time from full speed to both machine & 0.12 & 0.43 \\
\hline & & Switch time from both machine to cruise & 0.13 & 0.75 \\
\hline \multirow{5}{*}{ Concealment } & \multirow{5}{*}{0.09} & Underwater noise & 0.31 & 0.47 \\
\hline & & Aerodynamic noise & 0.26 & 0.39 \\
\hline & & Vibration intensity & 0.14 & 0.66 \\
\hline & & Infrared stealth & 0.21 & 0.75 \\
\hline & & Stealth performance & 0.08 & 0.55 \\
\hline \multirow{4}{*}{ Reliability } & \multirow{4}{*}{0.23} & Gas turbine reliability & 0.22 & 0.49 \\
\hline & & Line shafting reliability & 0.25 & 0.48 \\
\hline & & Propeller reliability & 0.28 & 0.77 \\
\hline & & gear box reliability & 0.25 & 0.81 \\
\hline \multirow{4}{*}{ Repairable } & \multirow{4}{*}{0.10} & Maintenance difficulty & 0.27 & 0.90 \\
\hline & & Spare parts supply & 0.32 & 0.87 \\
\hline & & Single part maintenance time & 0.21 & 0.56 \\
\hline & & Average pre-maintenance time & 0.20 & 0.51 \\
\hline
\end{tabular}




\subsection{Remark Set}

All possible results of assessment constitute remark set. As for this study, remark set is divided into 4 ranks such as excellence, fine, qualification, disqualification (see Equation (5)). 4 remark grades are transformed into the corresponding evaluation one (see Equation (6)).

$$
V=\left\{V_{1}, V_{2}, V_{3}, V_{4}\right\}
$$

In the equation: $V$-remark set;

$\boldsymbol{V}_{1}$-excellence, $90 \leq \mathrm{F}<100$;

$V_{2}$-fine, $75 \leq \mathrm{F}<90$;

$\boldsymbol{V}_{3}-$ qualification, $60 \leq \mathrm{F}<75$;

$\boldsymbol{V}_{4}$-disqualification, $\mathrm{F}<60$.

$$
\mathrm{F}=(90,75,60,50)^{T}
$$

\subsection{Evaluation Matrix}

The elements in Evaluation matrix indicate the degree of membership of Second-degree index, which can be obtained from the result of experts grading method. Equation (7) means the Evaluation matrix of economy. Similarly, $R_{i}(i=2,3,4,5)$ can be got.

$$
R_{1}=\left[\begin{array}{llll}
0.6 & 0.2 & 0.1 & 0.1 \\
0.5 & 0.3 & 0.1 & 0.1 \\
0.4 & 0.2 & 0.2 & 0.2 \\
0.3 & 0.2 & 0.2 & 0.3
\end{array}\right]
$$

\subsection{Fuzzy Comprehensive Evaluation}

The weight of first-degree estimate index:

$$
A=(0.34,0.24,0.09,0.23,0.10)
$$

The weight of second -degree estimate index:

$$
\begin{aligned}
& A_{1}=\{0.33,0.37,0.14,0.16\} \\
& A_{2}=\{0.24,0.23,0.28,0.12,0.13\} \\
& A_{3}=\{0.31,0.26,0.14,0.21,0.08\} \\
& A_{4}=\{0.22,0.25,0.28,0.25\} \\
& A_{5}=\{0.27,0.32,0.21,0.20\}
\end{aligned}
$$

The results of second -degree estimate are as follows:

$$
\begin{aligned}
& B_{1}=A_{1} \times R_{1}=(0.487,0.237,0.13,0.146) \\
& B_{2}=A_{2} \times R_{2}=(0.496,0.241,0.175,0.088) \\
& B_{3}=A_{3} \times R_{3}=(0.325,0.278,0.165,0.232) \\
& B_{4}=A_{4} \times R_{4}=(0.5,0.266,0.181,0.053) \\
& B_{5}=A_{5} \times R_{5}=(0.553,0.248,0.179,0.02)
\end{aligned}
$$

The final evaluation result of COGAG power plant performance, as show below:

$$
R=\left[\begin{array}{l}
B_{1} \\
B_{2} \\
B_{3} \\
B_{4} \\
B_{5}
\end{array}\right]=\left[\begin{array}{cccc}
0.487 & 0.237 & 0.13 & 0.146 \\
0.496 & 0.241 & 0.175 & 0.088 \\
0.325 & 0.278 & 0.165 & 0.232 \\
0.5 & 0.266 & 0.181 & 0.053 \\
0.553 & 0.248 & 0.179 & 0.02
\end{array}\right]
$$




$$
\begin{aligned}
& B=A \times R=(0.484,0.249,0.161,0.106) \\
& Z=B \times F=77.195
\end{aligned}
$$

The evaluation results: According to the principle of maximum membership degree, the overall goal of the general reviews is 77.195, and its evaluation level is fine.

\section{Conclusions}

The main conclusions of this work are the following:

- Results show that, the integrated index rank of this type was between excellence and fine which can guarantee the safety work of the ship. But the probability in the fine grade was higher which showed that the performance of this COGAG power plant should be improved and optimized.

- Comprehensive estimate of the power plant from the experts showed that the cover-up performance was worse. The box, shock isolation device and the coating absorbing technique could be used to the invisible goal. Shock isolation device was added in this system which could strengthen the damping of the mechanical stress wave so as to shortening the oscillation intensity.

- Proper parameter was chosen based on the comprehensive evaluation model on the performance of the COGAG which could quantity the performance index that is blurred. Then, reliable basis of design and demonstration of the power plant could be obtained.

- The principle and theory of the method could be used to estimate the performance index of other type of power plant.

The method could be translated and edited in computer program which can be used to the performance analysis of other kinds of power plant.

\section{References}

[1] Yu, Y.-H., Sun, F.-R. and Liu, Y.-B. (2008) Analysis of Load Efficiency Characteristics of COGAG Power Plant Based on Dynamic Simulation. Ship Engineering, 30.

[2] Gu, H.W. (2012) Research on Parallel Method of COGAG. Harbin Engineering University, Harbin.

[3] Altosole, M., Benvenuto, G., Figari, M. and Campora, U. (2009) Real-Time Simulation of a COGAG Naval Ship Propulsion System. Engineering for the Maritime Environment, 223, 47-61.

[4] Zadeh, L.A. (1965) Fuzzy Sets. Information and Control, 8, 338-353. http://dx.doi.org/10.1016/S0019-9958(65)90241-X

[5] Yang, L.-B. and Gao, Y.-Y. (2011) Principle and Application of Fuzzy Mathematics. South China University of Technology Press.

[6] Manuel, V., Dolores, D.M. and Antonio, R. (2003) Thermoeconomic Optimization of Combined Cycle Gas Turbine Power Plants Using Genetic Algorithms. Applied Thermal Engineering, 23, 2169-2182.

[7] Liang, Q.-C. and Zhang, S. (2011) Research on Fuzzy Judge Model of Ship Propulsion System. 2011 IEEE International Conference on Intelligent Computing and Integrated Systems, Gui Lin, 1, 451-453. 